\title{
AS MULHERES SÃO MENOS CITADAS DO QUE OS HOMENS EM ARTIGOS CIENTÍFICOS? UMA ANÁLISE DO COMPORTAMENTO DE CITAÇÃO RELACIONADO AO GÊNERO NAS PESQUISAS EM ETNOBIOLOGIA
}

\section{ARE WOMEN LESS QUOTED THAN MEN IN SCIENTIIIIC ARTICLES? AN ANALYSIS OF GENDER-RELATED CITATION BEHAVIOR IN ETHNOBIOLOGY RESEARCH}

Juliana Loureiro Almeida CAMPOS ${ }^{1}$, Andrêsa Suana Argemiro ALVES ${ }^{1}$, Flávia Rosa SANTORO

${ }^{1}$ Laboratório de Ecologia e Evolução de Sistemas Socioecológicos, Centro de Biociências, Departamento de Botânica, Universidade Federal de Pernambuco, Cidade Universitária, Recife, Pernambuco, Brasil.

*1oureiroju@hotmail.com

Submitted: 09/11/2020; Accepted: 20/01/2021; Published: 22/02/2021

\section{RESUMO}

A visibilidade de um artigo científico pode ser medida através do número de citações atribuído a ele, o qual pode ser influenciado por fatores como nacionalidade, autocitação, colaboração internacional, além de fatores relacionados ao gênero. Avaliamos se o comportamento de citação de artigos publicados em dois periódicos internacionais especializados em etnobiologia envolve um viés de gênero e se esse viés varia em relação ao continente da instituição de pesquisa e em relação à subárea de conhecimento etnobiológico. De forma geral, não encontramos diferenças significativas na média de citações recebidas em artigos publicados por homens e mulheres, porém, tanto as últimas como os primeiros citam significativamente mais homens em suas publicações. Independente do continente, homens são significativamente mais citados que mulheres. Autores do gênero masculino citam colegas do mesmo gênero em todas as subáreas, enquanto autoras do gênero feminino das subáreas etnobotânica, etnoecologia e etnozoologia citam significativamente mais autores do gênero masculino. Nossos resultados podem estar associados a um menor número de publicações assinadas por mulheres nos continentes asiáticos e africanos, assim como em todas as subáreas da etnobiologia. Além disso, outras questões já discutidas em estudos anteriores também se relacionam com o padrão de citação observado, como o fato de homens ocuparem maiores cargos de liderança, obterem maior prestígio e possuírem maior acesso a programas de fomento à pesquisa. Os resultados obtidos neste trabalho devem ser vistos como um instrumento, ainda que incipiente, de discussão e fomento para o subsídio de programas e políticas capazes de estabelecer condições igualitárias de acesso, permanência e desenvolvimento de mulheres na ciência etnobiológica.

PALAVRAS-CHAVE: Cientometria, Bibliometria, Produção científica, Publicações, Visibilidade.

\begin{abstract}
The visibility of a scientific article can be measured through the number of citations attributed to it, which can be influenced by many factors such as nationality, self-citation, international collaboration, in addition to factors related to gender. We assessed whether the behavior of citing articles published in two international journals specialized in ethnobiology involves a gender bias and whether this bias varies in relation to the continent of the research institution and in relation to the sub-area of ethnobiological knowledge. In general, we found no significant differences in the average number of citations received in articles published by men and women, but both the latter
\end{abstract}


CAMPOS, J. L. A.; ALVES, A. S. A.; SANTORO, F. R. As mulheres são menos citadas do que os homens em artigos científicos? Uma análise do comportamento de citação relacionado ao gênero nas pesquisas em etnobiologia. Ethnoscientia v.6 n. 2, especial, 2021. DOI: 10.22276/ethnoscientia.v6i2.371

and the former cite significantly more men in their publications. Regardless of the continent, men are significantly more cited than women. Male authors cite colleagues of the same gender in all subareas, while female authors in the sub-areas ethnobotany, ethnoecology and ethnozoology mention significantly more male authors. Such results may be associated with a smaller number of publications signed by women on Asian and African continents, as well as in all sub-areas of ethnobiology. In addition, other issues already discussed in previous studies are also related to the observed citation pattern, such as the fact that men occupy greater leadership positions, obtain greater prestige and have greater access to research promotion programs. The results obtained in this work must be seen as an instrument, albeit incipient, of discussion and promotion for the subsidy of programs and policies capable of establishing equal conditions of access, permanence and development of women in ethnobiological science.

KEYWORDS: Scientometrics, Bibliometrics, Scientific production, Publications, Visibility.

\section{INTRODUÇÃO}

A cientometria é definida como o estudo dos aspectos quantitativos das atividades científicas e tem sido utilizada para elaborar indicadores que possibilitam avaliar a produção científica de indivíduos, áreas de conhecimento e países (SILVA et al., 2011). O estudo do comportamento de citação faz parte da pesquisa cientométrica e tem se tornado cada vez mais relevante, já que o número de citações recebidas por um artigo científico pode indicar o nível de visibilidade e reconhecimento do mesmo (EYSENBACH, 2006).

Pesquisas têm evidenciado que o comportamento de citação pode ser influenciado por diversos fatores, como nacionalidade do primeiro autor, autocitação, insularidade, colaboração internacional, tipo de periódico que o artigo foi publicado, prestígio acadêmico e até mesmo fatores psicológicos e políticos (ALVARENGA, 1998; LORTIE et al., 2007; BORNMANN e DANIEL, 2008; GARGOURI et al., 2010; ALBUQUERQUE et al., 2013; SHAH et al. 2015; CAMPOS et al., 2016).

O gênero também é um fator que pode estar relacionado ao comportamento de citação (MALINIAK et al., 2013). De acordo com Larivière et al. (2013), artigos que possuem mulheres como primeiras autoras vinculadas a instituições localizadas em países com maiores índices de produção acadêmica foram menos citados que artigos publicados por homens nessas mesmas posições. Ao avaliarem as citações de artigos publicados na área de ciências políticas, Maliniak et al. (2013) verificaram que, de forma geral, primeiras autoras do gênero feminino são menos citadas que primeiros autores do gênero masculino e que os últimos tendem a citar mais pesquisadores homens do que mulheres. King et al. (2017) analisaram artigos indexados na base de dados JSTOR e observaram que pesquisadores homens publicaram mais artigos e apresentaram maior tendência de autocitação em comparação com pesquisadoras mulheres. Beaudry e Larivière (2016) 
CAMPOS, J. L. A.; ALVES, A. S. A.; SANTORO, F. R. As mulheres são menos citadas do que os homens em artigos científicos? Uma análise do comportamento de citação relacionado ao gênero nas pesquisas em etnobiologia. Ethnoscientia v.6 n. 2, especial, 2021. DOI: 10.22276/ethnoscientia.v6i2.371

observaram que pesquisadoras mulheres que possuem o mesmo número de coautores que pesquisadores homens ou que publicam em periódicos com fator de impacto semelhante são menos citadas que os últimos. Contrapondo esses achados, Cotropia e Petherbridge (2018) verificaram que artigos da área de legislação que possuem ao menos uma autora do gênero feminino são significativamente mais citados do que aqueles que não possuem nenhuma pesquisadora do gênero feminino como autora. Diante do exposto, é possível verificar que existe uma tendência de pesquisadores homens serem mais citados que pesquisadoras mulheres; no entanto, é importante destacar que essas diferenças de gênero podem variar entre áreas de pesquisa e entre os países cujas instituições de pesquisa estão vinculadas.

Com relação à literatura cientométrica relacionada ao campo da etnobiologia, estudos abordaram diferentes tópicos, por exemplo: se pesquisadores costumam relatar as contribuições teóricas dos trabalhos citados em suas pesquisas (RAMOS et al., 2012), traçaram um panorama dos trabalhos publicados por pesquisadores do Brasil e da América Latina (ALBUQUERQUE et al., 2013; RITTER et al., 2015), avaliaram a utilização do prefixo "etno" nas publicações (HANAZAKI, 2015), analisaram o grau de insularidade de artigos publicados (CAMPOS et al., 2016) e verificaram se pesquisas no campo da educação e etnobiologia fizeram articulações entre conhecimento científico e local (SOTERO et al., 2020). Apesar da literatura disponível, não foram localizados estudos que buscaram identificar diferenças de gênero no comportamento de citação de artigos publicados por pesquisadores dessa área.

Com a pretensão de avançar nessa discussão e contribuir com o avanço da pesquisa cientométrica no campo da etnobiologia, o presente estudo buscou avaliar se o comportamento de citação de artigos publicados em periódicos internacionais especializados na área de etnobiologia envolve um viés de gênero e se esse viés varia em relação ao continente da instituição de pesquisa e em relação à área de conhecimento etnobiológico.

\section{MATERIAL E MÉTODO}

\subsection{Coleta e organização de dados}

Analisamos todos os artigos científicos publicados nos anos de 2018 e 2019 em duas revistas científicas: Journal of Ethnobiology and Ethnomedicine (totalizando 148 artigos) e Ethnobiology and Conservation (totalizando 28 artigos). Escolhemos essas duas revistas por serem específicas do tema de etnobiologia, de acesso aberto em todo o mundo, indexadas 
CAMPOS, J. L. A.; ALVES, A. S. A.; SANTORO, F. R. As mulheres são menos citadas do que os homens em artigos científicos? Uma análise do comportamento de citação relacionado ao gênero nas pesquisas em etnobiologia. Ethnoscientia v.6 n. 2, especial, 2021. DOI: 10.22276/ethnoscientia.v6i2.371

em bases de dados internacionais e que possuem publicações de autores de diversos continentes.

Para cada artigo, verificamos o gênero e o continente da instituição de vínculo dos primeiros autores, a subárea dentro da etnobiologia, o número de vezes que o artigo foi citado e o número de artigos incluídos nas referências bibliográficas. O gênero dos primeiros autores foi verificado através do primeiro nome dos autores, quando era evidente, ou através da busca em plataformas como ResearchGate (https://www.researchgate.net/) para a visualização da foto de cada autor. Nesse caso, consideramos apenas o gênero identificado pela foto. Para quatro autores não pudemos encontrar fotos ou qualquer indício do gênero do autor a partir do primeiro nome, portanto, esses quatro artigos foram excluídos de nossas análises.

O continente dos primeiros autores de cada artigo foi acessado através da instituição de vínculo identificada nos artigos. Para identificar as subáreas da etnobiologia, utilizamos a classificação de Albuquerque et al. (2013), a partir da leitura dos títulos, resumos e palavras-chave de cada artigo. Dessa forma, os artigos amostrados foram classificados nas subáreas etnobiologia, etnobotânica, etnozoologia, etnoecologia e etnomedicina. Para verificar quantas vezes cada artigo foi citado, utilizamos a ferramenta da base de dados do Google Scholar, que aponta o número de citação recebida por cada artigo.

Para identificar o gênero dos primeiros autores das publicações citadas nos artigos incluídos no nosso banco de dados, selecionamos 10 referências bibliográficas de cada artigo através de amostragem aleatória sem substituição, a partir do número total de referências citadas. A seleção aleatória foi realizada com o programa BioEstat 5.3 (AYRES et al., 2007). Com a ajuda da base de dados do Google Scholar, buscamos todos os artigos selecionados nas referências bibliográficas e classificamos o gênero dos autores como feminino ou masculino a partir do primeiro nome, quando evidente, ou a partir da foto em plataformas como o ResearchGate. Dessa forma, nosso banco de dados foi organizado da seguinte maneira:

- Nome da revista científica;

- Ano de publicação;

- Título do artigo;

- Área de conhecimento que classificamos como etnobiologia, etnobotânica, etnoecologia, etnozoologia e etnomedicina;

- Gênero do primeiro autor;

- Continente da instituição de vínculo do primeiro autor; 
CAMPOS, J. L. A.; ALVES, A. S. A.; SANTORO, F. R. As mulheres são menos citadas do que os homens em artigos científicos? Uma análise do comportamento de citação relacionado ao gênero nas pesquisas em etnobiologia. Ethnoscientia v.6 n. 2, especial, 2021. DOI: 10.22276/ethnoscientia.v6i2.371

- Número de vezes que o artigo foi citado;

- Número de vezes que o artigo citou uma primeira autora mulher (dentro dos 10 artigos das referências selecionados aleatoriamente);

- Número de vezes que o artigo citou um primeiro autor homem (dentro dos 10 artigos das referências selecionados aleatoriamente).

Ao total, foram analisados 172 artigos, 96 cujos primeiros autores eram homens e 76 nos quais as primeiras autoras eram mulheres. Entre esses, 144 artigos foram publicados pelo periódico Journal of Ethnobiology and Ethnomedicine, sendo 80 deles divulgados no ano de 2018 e 64 no em 2019; além de 28 artigos da revista Ethnobiology and Conservation, sendo 15 publicados em 2018 e 13 no ano subsequente de 2019.

\section{ANÁLISE DE DADOS}

Para conhecer a distribuição dos nossos dados, realizamos o teste Lilliefors de normalidade. A distribuição dos dados obtidos nessa pesquisa foi não paramétrica e, portanto, utilizamos o teste de Mann-Whitney em todas as análises estatísticas, as quais foram realizadas por meio do Software R 3.5.3 (R DEVELOPMENT CORE TEAM, 2015) e BioEstat 5.3 (AYRES et al., 2007).

Para verificar se existiam diferenças significativas entre o número médio de citações recebidas nos artigos publicados por autores homens e autoras mulheres, comparamos o número médio de citações recebidas para cada um dos gêneros. Para identificar se existiam diferenças de gênero nas citações incluídas nos artigos analisados, separamos os artigos produzidos por primeiros autores homens e primeiras autoras mulheres e verificamos a existência de diferenças significativas no número médio de artigos produzidos por homens e mulheres incluídos nas referências bibliográficas.

Para identificar diferenças entre os continentes, igualmente separamos as análises primeiramente entre primeiras autoras mulheres, comparando a média de artigos produzidos por autores homens e mulheres incluídos nas referências bibliográficas para cada continente. Analisamos somente os continentes América, África, Ásia e Europa porque houve apenas dois artigos cuja instituição de vínculo das primeiras autoras era Oceania. Inicialmente também pensamos em subdividir o continente americano, dado sua diversidade social, econômica e cultural, o que influenciaria o comportamento de citação, no entanto, ao se fazer essa divisão, o n para a América Central (1) e América do Norte (4) se reduziram muito. Dessa forma, decidimos por unificar em uma mesma análise todos os estudos do continente americano. Fizemos o mesmo para os primeiros autores homens, analisando os 
CAMPOS, J. L. A.; ALVES, A. S. A.; SANTORO, F. R. As mulheres são menos citadas do que os homens em artigos científicos? Uma análise do comportamento de citação relacionado ao gênero nas pesquisas em etnobiologia. Ethnoscientia v.6 n. 2, especial, 2021. DOI: 10.22276/ethnoscientia.v6i2.371

mesmos continentes, pois a representatividade da Oceania também foi baixa (um artigo), e unindo América do Norte (sete artigos) e América Central (nenhum artigo) com todos os artigos da América do Sul.

Para verificar diferenças de gênero nas citações dos artigos entre as subáreas da etnobiologia, também analisamos primeiro entre as primeiras autoras mulheres, comparando a média do número de referências (incluídas nesses artigos) produzidos por autores homens e mulheres para cada subárea: etnobiologia, etnoecologia, etnobotânica, etnozoologia e etnomedicina. Fizemos o mesmo para os artigos de primeiros autores homens. Os resultados das análises estatísticas podem ser verificados no material suplementar (Anexo-Tabela 1).

\section{RESULTADOS}

\subsection{Diferenças no número de citações recebidas por gênero de autoria}

Não foram encontradas diferenças significativas no número de citações recebidas nos artigos publicados por mulheres e homens $(\mathrm{W}=3367 ; \mathrm{p}=0,38)$. Esse resultado aponta que nas publicações científicas em etnobiologia, pesquisadores do gênero masculino e feminino recebem uma média de citações semelhante.

\subsection{Diferenças de gênero nas citações incluídas nos artigos de forma geral}

Obtivemos diferenças significativas para autoras mulheres $(W=1110.5 ; p=<0.001)$ e para autores homens $(\mathrm{W}=403.50 ; \mathrm{p}=<0.001)$, sendo que, em ambos os casos, os artigos analisados utilizam majoritariamente autores do gênero masculino em suas fundamentações científicas.

\subsection{Diferenças de gênero nas citações incluídas nos artigos por continente}

A Figura 1 exibe o número de artigos científicos redigidos por primeiros autores do gênero masculino e primeiras autoras do gênero feminino em cada continente analisado. 
CAMPOS, J. L. A.; ALVES, A. S. A.; SANTORO, F. R. As mulheres são menos citadas do que os homens em artigos científicos? Uma análise do comportamento de citação relacionado ao gênero nas pesquisas em etnobiologia. Ethnoscientia v.6 n. 2, especial, 2021. DOI: 10.22276/ethnoscientia.v6i2.371

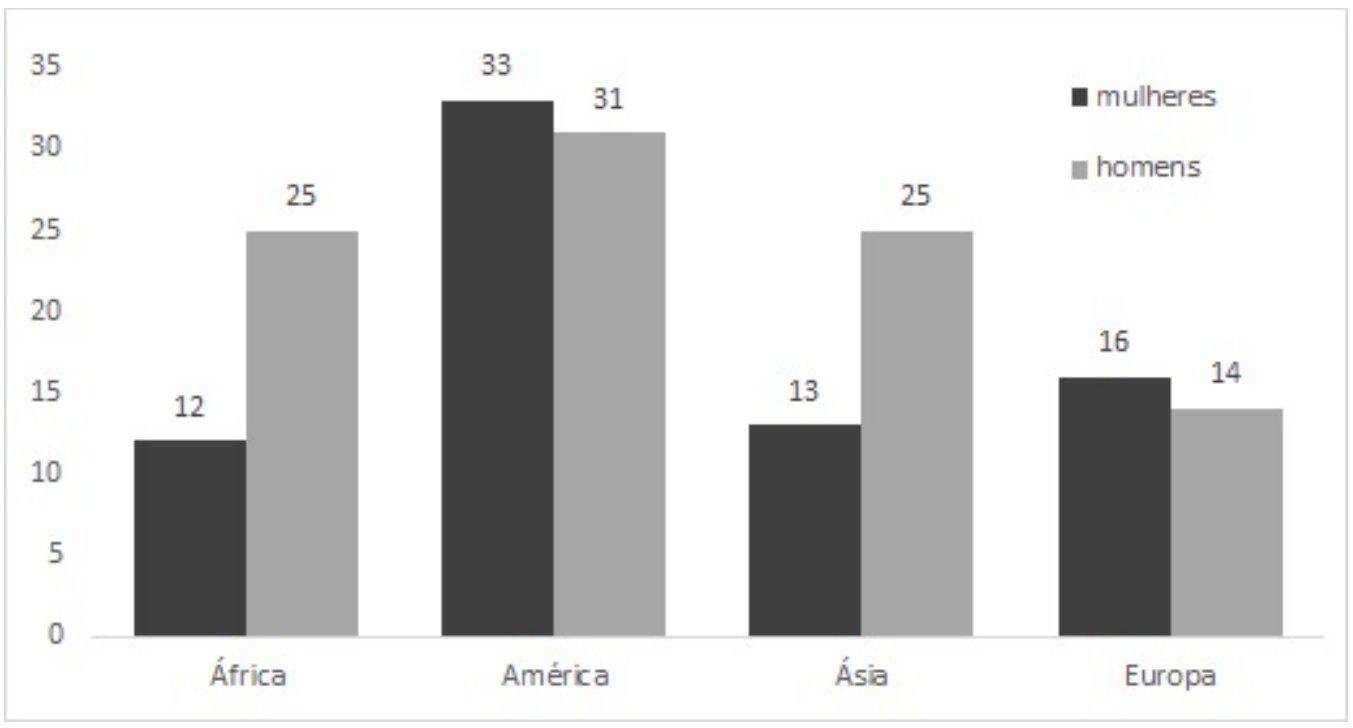

Figura 1: Número de artigos científicos produzidos por primeiros autores do gênero masculino e primeiras autoras do gênero feminino em cada continente, publicados nos anos de 2018 e 2019 nas revistas científicas Journal of Ethnobiology and Ethnomedicine e Ethnobiology and Conservation. Fonte: autoria própria.

Com relação às diferenças de gênero nas citações incluídas nos artigos analisados por continente, obtivemos diferenças significativas quando observado o comportamento dos autores do gênero masculino, no qual foi revelado maior número de citações para trabalhos publicados por pesquisadores do mesmo gênero, sendo: $\mathrm{W}=0.50$ e $\mathrm{p}=<0.001$ para autores africanos; $\mathrm{W}=28.5$ e $\mathrm{p}=<0.001$ para autores asiáticos; $\mathrm{W}=0.50$ e $\mathrm{p}=<0.001$ para autores europeus; $\mathrm{W}=98.0$ e $\mathrm{p}=<0.001$ para autores americanos.

A mesma análise feita para verificação do padrão de citação dos artigos produzidos por pesquisadoras do gênero feminino por continente também evidenciou significativa predominância de citação de autores homens, indicando os seguintes valores: $\mathrm{W}=9.00 \mathrm{e}$ $\mathrm{p}=<0.01$ para autoras africanas; $\mathrm{W}=162.50$ e $\mathrm{p}=<0.001$ para autoras asiáticas; $\mathrm{W}=52.00$ e $\mathrm{p}<0.01$ para autoras europeias; $\mathrm{W}=178.00 \mathrm{e} \mathrm{p}=<0.001$ para autoras americanas.

\subsection{Diferenças de gênero nas citações incluídas nos artigos por subárea de conhecimento dentro da etnobiologia}

A Figura 2 exibe o número de artigos científicos redigidos por primeiros autores do gênero masculino e primeiras autoras do gênero feminino em cada subárea da etnobiologia. 
CAMPOS, J. L. A.; ALVES, A. S. A.; SANTORO, F. R. As mulheres são menos citadas do que os homens em artigos científicos? Uma análise do comportamento de citação relacionado ao gênero nas pesquisas em etnobiologia. Ethnoscientia v.6 n. 2, especial, 2021. DOI: 10.22276/ethnoscientia.v6i2.371

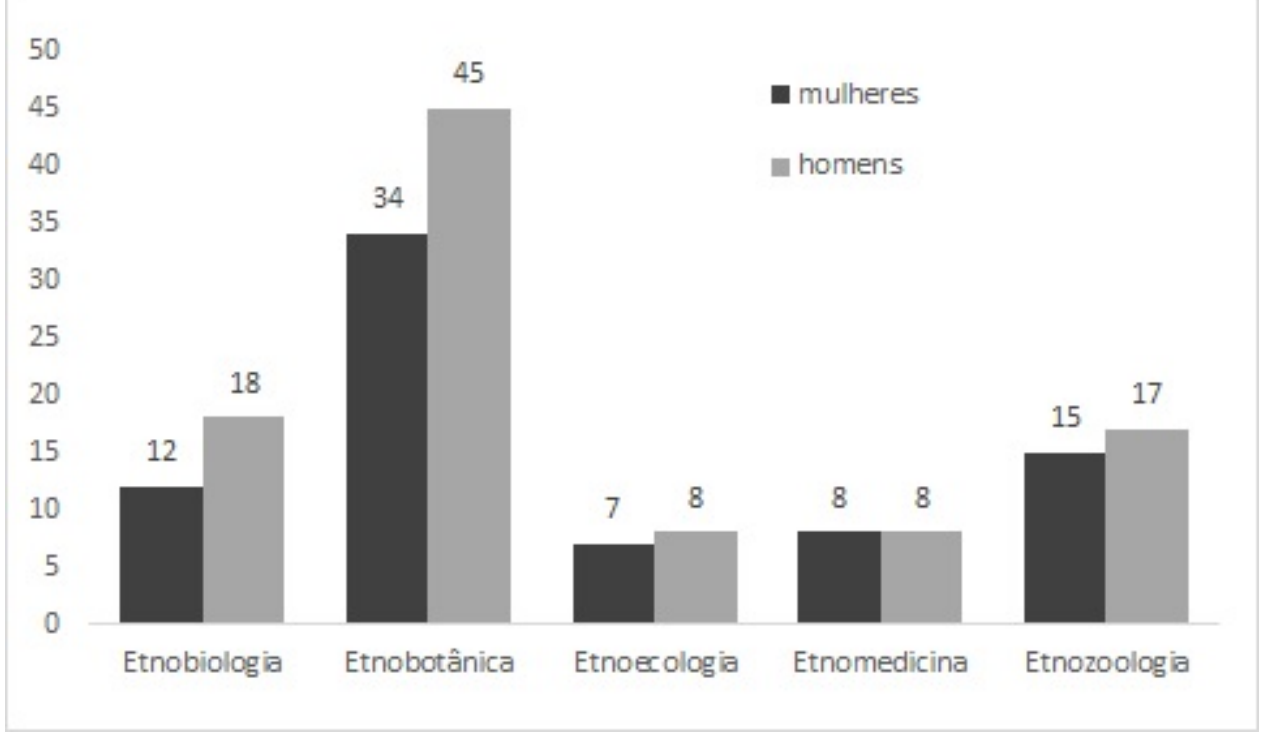

Figura 2: Número de artigos científicos produzidos por primeiros autores do gênero masculino e primeiras autoras do gênero feminino em cada subárea da etnobiologia, publicados nos anos de 2018 e 2019 nas revistas científicas Journal of Ethnobiology and Ethnomedicine e Ethnobiology and Conservation. Fonte: autoria própria.

Analisamos a possível existência de diferenças de gênero nas citações incluídas nos artigos de acordo com as subáreas da etnobiologia e obtivemos os seguintes resultados: para autores do gênero masculino, existem diferenças significativas no padrão de citação por subárea, uma vez que o maior número de citação é também para homens $(\mathrm{W}=33.50$ e $p=<0.001$ na etnobiologia; $W=106.50$ e $p=<0.001$ na etnobotânica; $W=0.50$ e $p<0.001$ na etnoecologia; $\mathrm{W}=2.00$ e $\mathrm{p}<0.001$ na etnomedicina; $\mathrm{W}=0.00$ e $\mathrm{p}=<0.001$ na etnozoologia).

Com relação às diferenças de gênero nas citações feitas por pesquisadoras mulheres das diferentes subáreas da etnobiologia, encontramos dois diferentes padrões, os quais se revelaram por subáreas. Sendo assim, temos como primeiro padrão a ausência de diferenças significativas nas citações para autoras mulheres da subárea etnobiologia ( $W=39.50$ e $\mathrm{p}=0.06)$ e da etnomedicina $(\mathrm{W}=30.00$ e $\mathrm{p}=0.8336)$. O segundo padrão caracteriza-se por trabalhos realizados por mulheres que citam significativamente mais homens nas seguintes subáreas, respectivamente: $\mathrm{W}=196.00$ e $\mathrm{p}=<0.001$ para autoras mulheres da subárea etnobotânica; $\mathrm{W}=9.0$ e $\mathrm{p}<0.05$ da etnoecologia; $\mathrm{W}=3.7952$ e $\mathrm{p}<0.001$ da etnozoologia.

\section{DISCUSSÃO}

O número de citações recebidas por um artigo científico consiste em um dos diversos indicadores bibliométricos usados em cientometria. Esses indicadores se baseiam geralmente em quantidade e são utilizados para diversos fins, como para a concessão de 
CAMPOS, J. L. A.; ALVES, A. S. A.; SANTORO, F. R. As mulheres são menos citadas do que os homens em artigos científicos? Uma análise do comportamento de citação relacionado ao gênero nas pesquisas em etnobiologia. Ethnoscientia v.6 n. 2, especial, 2021. DOI: 10.22276/ethnoscientia.v6i2.371

fomento às instituições de pesquisa e projetos vinculados às mesmas, bem como para avaliar o impacto do trabalho realizado por pesquisadores (Hohendorff et al., 2016).

Nossos resultados sugerem que o comportamento de citação entre pesquisadores na etnobiologia apresenta variações a depender da amplitude da pergunta, entretanto, nessa análise, fica evidente que há um padrão de maior número de citações recebidas para artigos em que a primeira autoria pertence a pesquisadores do gênero masculino em relação àqueles escritos por pesquisadoras do gênero feminino.

\subsection{Diferenças no número de citações recebidas por gênero de autoria}

Ao contrário do que mostram os demais resultados, não encontramos diferenças significativas entre o número de citações recebidas por artigos cujo primeiro autor é homem em comparação com aqueles cuja primeira autora é mulher. Uma explicação para que esse resultado seja diferente dos outros padrões observados é que o período em questão possivelmente não permita avaliar de maneira precisa a influência do gênero nos padrões de citação, uma vez que grande parte dos artigos analisados nesta pesquisa, em especial aqueles com publicação no último ano, apresentavam uma ou nenhuma citação relacionada. Esse fato pode ser justificado pelo tempo percorrido entre submissão, aceite e/ou publicação dos artigos subsequentes à divulgação daqueles avaliados. Isto é, após publicação de determinado trabalho científico, os artigos que, porventura, poderiam citá-lo, precisam também passar por seus próprios processos de avaliação por pares e publicação em meio de divulgação científica. Nesse sentido, Hohendorff et al. (2016) realizaram uma investigação na qual apontam que, nos periódicos brasileiros da área de Psicologia, ciência que é frequentemente relacionada ao papel de cuidadora designado às mulheres, a depender do Qualis da revista em que se pretende publicar, há um hiato que varia, em média, entre 8 e 12 meses entre a data de submissão até a data de aceite, sendo esse tempo diretamente proporcional aos mais altos estratos Qualis das revistas. Logo, é possível que estudos que contenham em seus bancos de dados artigos com maior tempo de publicação, possam apresentar resultados diferentes desses aqui apresentados.

\subsection{Diferenças de gênero nas citações incluídas nos artigos por continente}

Nossos resultados apontaram que tanto homens quanto mulheres citam mais trabalhos produzidos por homens nas suas publicações, independente do continente do primeiro autor/ primeira autora do artigo. Quando vista de maneira global, a participação das mulheres em carreiras relacionadas à ciência e tecnologia, sobretudo nas instituições de 
CAMPOS, J. L. A.; ALVES, A. S. A.; SANTORO, F. R. As mulheres são menos citadas do que os homens em artigos científicos? Uma análise do comportamento de citação relacionado ao gênero nas pesquisas em etnobiologia. Ethnoscientia v.6 n. 2, especial, 2021. DOI: 10.22276/ethnoscientia.v6i2.371

ensino superior, teve significativo aumento a partir da década de 1970 em países da América Latina, Ásia e Europa Ocidental, embora esse acesso tenha sido relativamente mais lento em alguns países do continente africano (LETA, 2003). Ainda que consideremos esse avanço relativamente recente na história da ciência, de maneira geral, as mulheres representam uma minoria entre a categoria de pesquisadores em todo o mundo, compondo apenas $28 \%$ da população de pessoas empregadas nas áreas de pesquisa e desenvolvimento (UNESCO, 2018; WHO, 2020).

Apesar da grande demanda para que se produzam dados estatísticos comparáveis entre os países e as mulheres cientistas, esses dados, bem como o uso dos mesmos na elaboração de políticas públicas que visem maior participação feminina na ciência, são ainda bastante limitados (UNESCO, 2018). Com relação às análises direcionadas à compreensão do padrão de citação por continente, nossos resultados apontam que, apesar da maior profusão experienciada por mulheres na academia, os maiores índices de citação ainda estão voltados àqueles estudos assinados por homens em todos os continentes considerados em nossas análises. Sendo assim, ainda que se considerem as distintas condições socioeconômicas e culturais nos diversos continentes do globo e os fatores que influenciam fortemente a participação das mulheres na ciência, o padrão observado no comportamento de citação não difere quando comparados territórios em que mulheres apresentam maior ou menor acesso à participação na produção científica.

$\mathrm{Na}$ América Latina, o Brasil é o país que concentra o maior percentual de mulheres em assinaturas de artigos científicos com cerca de $72 \%$ de toda a produção científica nacional, incluindo ao menos uma mulher na autoria (ALBORNOZ et al., 2018). Em sequência, Argentina e Guatemala seguem, respectivamente, com 67\% e 66\% das publicações científicas com participação feminina. Ainda de acordo com esse relatório, no ano de 2017, as mulheres foram responsáveis por cerca de 53\% dos estudos científicos publicados no Brasil, enquanto os homens assinaram 47\% das publicações. No Brasil, embora o perfil típico dos docentes das instituições de ensino superior seja masculino (BRASIL, 2019), também nesse período houve um crescimento da participação das mulheres na academia não apenas na qualidade de estudantes, mas também na posição de docentes e pesquisadoras (LETA, 2003). É nesse cenário que a etnobiologia tem avançado, não apenas no contexto quantitativo, mas, sobretudo, na contribuição teórica que os diversos estudos liderados por pesquisadores na América Latina, em especial Brasil, Argentina e México, têm oferecido para o desenvolvimento da disciplina (ALBUQUERQUE et al., 2013; SILVA et al., 2019). 
CAMPOS, J. L. A.; ALVES, A. S. A.; SANTORO, F. R. As mulheres são menos citadas do que os homens em artigos científicos? Uma análise do comportamento de citação relacionado ao gênero nas pesquisas em etnobiologia. Ethnoscientia v.6 n. 2, especial, 2021. DOI: 10.22276/ethnoscientia.v6i2.371

Talvez devido à sua natureza interdisciplinar, a etnobiologia aparente ser uma disciplina de caráter mais integrador na qual a participação de ambos os gêneros em sua construção é incentivada. No entanto, essa perspectiva pode ser confrontada quando observadas as diversas questões que perpassam pelas dimensões de gênero, como aquelas levantadas por Silva et al. (2019) em investigação que buscou compreender os desafios e perspectivas das pesquisadoras em etnobiologia no Brasil, país que lidera a produção científica da etnobiologia mundial. As autoras encontraram resultados interessantes, que à primeira vista podem colocar a etnobiologia como uma área progressista dentro das ciências da natureza, como a similaridade no número de publicações assinadas por homens e mulheres como autores principais nos artigos científicos da área; no entanto, foi visto também que a maioria dos líderes de pesquisa em etnobiologia pertence ao gênero masculino e que estes também conseguem publicar em periódicos de maior impacto. Nesse sentido, é possível que, na pesquisa etnobiológica, mesmo em contextos como o do Brasil, homens tenham maior acesso a programas de incentivo à pesquisa devido às posições de liderança e que também sejam mais citados, visto que além do fato de que altas posições hierárquicas geram maior prestígio profissional, o que aumenta as chances de citação, as publicações em periódicos de alto impacto também são consideradas como vieses no padrão de citação (ver VANZ e CAREGNATO, 2003; RAMOS et al., 2012).

Sobre o continente europeu, no final do séc. XX, a ideia de manutenção dos centros acadêmicos como locais de poder e privilégios masculinos começou a ser rechaçada, pois foi considerada como violação dos direitos humanos (OSBORN, 1994; GALINDO, 2016). Portanto, para que houvesse uma mudança nesse panorama ao longo dos anos subsequentes, em especial desde a década de 1990, programas começaram a ser desenvolvidos para esse fim através da União Europeia (UE), que adotou abordagens estratégicas básicas para fomentar políticas públicas sensíveis às desigualdades de gênero e necessidades das mulheres (GALINDO, 2016). Assim, a igualdade de gênero nas áreas de investigação e inovação tornou-se prioridade para a UE, sendo também um compromisso a revisão e renovação dessas estratégias a cada cinco anos (GALINDO, 2016; EUROPEAN UNION, 2019). Tais esforços apontam que atualmente há uma tendência de crescimento no número de mulheres que trabalham com ciência na maioria dos países europeus, no entanto, elas ainda são minoria nas ocupações desses cargos (EUROPEAN UNION, 2019), principalmente em altas posições da hierarquia científica (OSBORN, 1994). Dessa maneira, ainda que haja programas que visem à diminuição das desigualdades entre gêneros em diversos países do continente europeu, em especial aqueles membros da UE, é necessário 
CAMPOS, J. L. A.; ALVES, A. S. A.; SANTORO, F. R. As mulheres são menos citadas do que os homens em artigos científicos? Uma análise do comportamento de citação relacionado ao gênero nas pesquisas em etnobiologia. Ethnoscientia v.6 n. 2, especial, 2021. DOI: 10.22276/ethnoscientia.v6i2.371

que sejam aumentados os esforços nesse sentido, pois muitas variáveis influenciam os padrões de citação, entre elas, o prestígio dos autores, que é sabidamente maior entre pesquisadores que ocupam posições mais altas na hierarquia da ciência, como mencionado acima.

$\mathrm{Na}$ Ásia, até 30 anos passados, era comum que mulheres que tinham interesse em seguir uma carreira científica saíssem de seus países de origem rumo à Europa e EUA para obter educação e formação em ciência (IP, 2011). Dessa forma, a migração que essas jovens pesquisadoras realizavam, muitas vezes, estava relacionada às menores chances que países asiáticos ofereciam para que elas superassem barreiras em relação ao gênero devido aos obstáculos impostos pela cultura tradicional (IP, 2011). Atualmente, devido ao desenvolvimento de políticas governamentais visando ao desenvolvimento da ciência e tecnologia com enfoque na educação, treinamento e oportunidades no setor profissional, diversos países no continente asiático estão no mesmo nível de inovação, produção e avanços tecnológicos que os EUA e Europa. Nesse sentido, Ip (2011) aponta que atualmente existem consideráveis oportunidades para jovens cientistas, sobretudo nas áreas de biociências.

Apesar do avanço observado em países do leste asiático, um estudo realizado por Chaudhuri (2009) aponta que essa realidade não é compartilhada com aqueles que integram a porção sul e sudeste da Ásia. Atualmente, alguns países da região sul da Ásia estão entre os que apresentam as mais baixas taxas de emprego e remuneração para as mulheres no mundo e diversos estudos apontam que a situação tende a se agravar. Em algumas localidades, como observado na Índia, a participação das mulheres no trabalho está em declínio (NARAYAN e SHARMILA, 2019), uma vez que se espera que as mulheres desempenhem apenas papéis voltados à vida doméstica, enquanto dos homens, espera-se e incentiva-se espaços de poder como cargos públicos e políticos. Além de tímida participação econômica que muitas mulheres viventes em países dessa região apresentam, elas também sofrem com baixíssimos níveis de escolaridade e estão entre aquelas com maiores taxas de fecundidade (CHAUDHURI, 2009). Em países mais autoritários, como o Butão, diversos direitos humanos básicos são negados às mulheres, incluindo o acesso à educação, além da discriminação e da exploração sexual serem comuns (ABBAS e MUNEER, 2019). Nossos resultados mostram que, além da baixa citação de mulheres nesse continente, há uma baixa participação de mulheres como primeiras autoras dos artigos analisados, muito menos representativa que em outros continentes, apenas comparável à África. 
CAMPOS, J. L. A.; ALVES, A. S. A.; SANTORO, F. R. As mulheres são menos citadas do que os homens em artigos científicos? Uma análise do comportamento de citação relacionado ao gênero nas pesquisas em etnobiologia. Ethnoscientia v.6 n. 2, especial, 2021. DOI: 10.22276/ethnoscientia.v6i2.371

No continente africano as desigualdades entre os gêneros, o acesso às carreiras científicas, bem como a permanência das mulheres nas áreas de ciência e tecnologia são ainda mais difíceis de serem compreendidas e, consequentemente, mitigadas. Essa dificuldade encontrada pelas mulheres cientistas se dá principalmente pelo fato de que o ainda forte enraizamento do patriarcado no continente pode afetar a função e o status das mulheres, resultando em divisões marcantes de gênero nas questões relacionadas ao trabalho tanto nas famílias quanto nas instituições (PROZESKY e MOUTON, 2019). Esses desafios, associados àqueles tradicionalmente apoiados nas questões de gênero como o cuidado com as crianças e as tarefas domésticas, têm o potencial de afetar negativamente o desempenho das mulheres cientistas, em especial àquelas que se encontram em situação emergente (TAMALE e OLOKA-ONYANGO, 1997; PROZESKY e MOUTON, 2019). Mesmo tendo que lidar com todos esses desafios para perseguir seus interesses acadêmicos enquanto cumprem as obrigações tipicamente delegadas às mulheres, a academia julga em pé de igualdade homens e mulheres ao considerar questões como produção e competência (TAMALE e OLOKA-ONYANGO, 1997). Para Okeke et al. (2017), a participação das mulheres nas universidades em muitos países africanos tem aumentado nas áreas de saúde, humanidades, comércio e educação, no entanto, nas áreas relacionadas às ciências da natureza, desde a graduação até os níveis de pós-graduação, a participação feminina nos últimos anos apresentou queda. Para esses autores, além das questões relacionadas ao trabalho doméstico realizado tradicionalmente por mulheres, existem ainda outros fatores que podem contribuir com o afastamento das mulheres nas ciências da natureza, entre os quais figuram a discriminação aberta e o forte desencorajamento dispensado às mulheres que se interessam por essa área.

Vale salientar ainda que a escassez de estudos voltados à temática sobre questões de gênero em diversos países africanos, muitas vezes fruto da relutância de acadêmicos do gênero masculino - pois são eles os que dominam os órgãos de ensino e pesquisa - se reflete em uma grande lacuna sobre dados capazes de evidenciar os desafios encontrados por mulheres cientistas nos diversos países do continente (TAMALE e OLOKAONYANGO, 1997). Sendo assim, muitas das questões apresentadas nos estudos sobre a participação das mulheres na ciência nos países do continente africanos baseiam-se no conhecimento empírico como uma importante ferramenta para levantar discussões e alcançar sucesso no desenvolvimento da ciência e tecnologia na África (TAMALE e OLOKA-ONYANGO, 1997; PROZESKY e MOUTON, 2019). Apesar da ausência de dados mais robustos sobre produção científica realizada por mulheres africanas, e de que 
CAMPOS, J. L. A.; ALVES, A. S. A.; SANTORO, F. R. As mulheres são menos citadas do que os homens em artigos científicos? Uma análise do comportamento de citação relacionado ao gênero nas pesquisas em etnobiologia. Ethnoscientia v.6 n. 2, especial, 2021. DOI: 10.22276/ethnoscientia.v6i2.371

claramente existe uma representatividade menor de mulheres como primeiras autoras segundo nossos dados, os resultados encontrados para número de citações nesse continente não diferem do que foi observado para as mulheres dos demais continentes analisados. Dessa forma, por mais que estudos cientométricos apontem que a nacionalidade dos autores seja um dos fatores mais influentes quando considerados os padrões de citação na etnobiologia (VANZ e CAREGNATO, 2003; RAMOS et al., 2012), é muito possível que, de acordo com os dados encontrados nesta pesquisa, o gênero tenha uma influência ainda mais forte e determinante nos padrões de citação.

Isso evidencia que a dificuldade encontrada por mulheres para ingressar, se manter e, sobretudo, se destacar nas carreiras científicas esbarra em fatores de caráter sistêmico e global. Apesar de perpassar por questões socioculturais características de cada continente, que são altamente diversas entre e dentro dos países, a maioria das mulheres lida com a sobrecarga do trabalho doméstico, as diversas questões relacionadas à maternidade e, não menos importante, o grande preconceito sofrido por seus próprios pares, bem como da sociedade em geral. Dessa maneira, é muito possível que o fato de as mulheres serem menos citadas do que homens, independentemente do continente de sua origem, passe também por questões como posições de liderança e prestígio, variáveis que na etnobiologia não discordam do cenário vigente. Se há uma correlação negativa entre a participação das mulheres na ciência com os mais altos cargos na hierarquia científica, é razoável supor que mulheres pesquisadoras gozem de menor prestígio profissional que os homens e, consequentemente, a popularidade de suas publicações pode sofrer essa influência.

\subsection{Diferenças de gênero nas citações incluídas nos artigos por subárea de conhecimento dentro da Etnobiologia}

Seguindo o padrão observado em todas as análises consideradas nesta investigação em relação às subáreas da etnobiologia, autores do gênero masculino também citam mais seus pares genéricos do que mulheres. Fato que se repete no comportamento de citação de autoras do gênero feminino para as subáreas de etnobotânica, etnoecologia e etnozoologia. Para Silva et al. (2019), algumas subáreas em etnobiologia apresentam uma maior concentração de pesquisadores do gênero masculino. Segundo as autoras, isso se dá por diversos filtros socioculturais arraigados na disciplina ou atuação na mesma, que desafiam ou mesmo atuam como impeditivos da participação das mulheres quando consideradas áreas específicas como a etnozoologia. Nessa subárea o grupo de informantes é, na maior parte dos casos, formado apenas por homens, que a depender das suas crenças podem não 
CAMPOS, J. L. A.; ALVES, A. S. A.; SANTORO, F. R. As mulheres são menos citadas do que os homens em artigos científicos? Uma análise do comportamento de citação relacionado ao gênero nas pesquisas em etnobiologia. Ethnoscientia v.6 n. 2, especial, 2021. DOI: 10.22276/ethnoscientia.v6i2.371

permitir a presença de mulheres nos eventos de caça e pesca. Dessa maneira, grande parte do grupo de pesquisadores é também masculina. É possível que esse dado, para além da receptividade nas atividades de campo, tenha início na educação básica e seja reforçado na academia a partir da ideia de que indivíduos do gênero masculino apresentam maior desenvoltura para atividades científicas, bem como para aquelas que contenham algum grau de esforço físico.

Áreas como etnozoologia, etnoecologia e etnobotânica muitas vezes envolvem métodos de coleta de dados biológicos que exigem, em maior ou menor grau, certo esforço físico dos participantes da pesquisa. É muito comum que pesquisadoras em etnobiologia enfrentem constantemente situações em que os colegas do gênero masculino sugerem que mulheres apresentam incapacidade moral e física (SILVA et al., 2019) e tais agressões podem influenciar diretamente na participação das mulheres em investigações dessa magnitude. Ainda no trabalho publicado por Silva et al. (2019), as autoras apontam para um dado bastante interessante, evidenciando que no Brasil, apesar de o número de publicações não diferir entre autores do gênero feminino ou masculino, os últimos são maioria entre líderes de pesquisa e também publicam seus artigos em periódicos de mais alto impacto em comparação com as primeiras. Junto a isso, sabe-se que as subáreas de etnoecologia e etnobotânica, sobretudo a última, consistem nas áreas em que os estudos etnobiológicos mais avançaram, incluindo aí questões relacionadas ao desenvolvimento teórico da disciplina. Essas áreas historicamente apresentam homens como líderes de pesquisa, portanto, é possível que variáveis como apoio institucional, financeiro, bem como o prestígio influenciem o comportamento de citação para essas subáreas (ALBUQUERQUE et al., 2011).

Para as subáreas de etnobiologia e etnomedicina, a ausência de diferenças significativas no número de citação entre os gêneros nos trabalhos liderados por mulheres certamente está relacionada ao fato de que nessas subáreas podemos encontrar uma gama de investigações relacionadas a estudos de comportamento, educação, saúde e cuidado, temas historicamente relacionados ao papel da mulher. Nesse sentido, é razoável pensar que o acesso das mulheres a essas subáreas e a manutenção dessas em instituições ou grupos de pesquisas voltados a esses temas podem ser, ainda que minimamente, favoráveis à conciliação das atividades de pesquisa, às exigências de publicação e ao acúmulo de funções que se somam com as atividades domésticas. Não menos importante, esse dado pode representar um nicho de ocupação profissional no qual parte dos pesquisadores do gênero masculino em etnobiologia não tem notório interesse. 
CAMPOS, J. L. A.; ALVES, A. S. A.; SANTORO, F. R. As mulheres são menos citadas do que os homens em artigos científicos? Uma análise do comportamento de citação relacionado ao gênero nas pesquisas em etnobiologia. Ethnoscientia v.6 n. 2, especial, 2021. DOI: 10.22276/ethnoscientia.v6i2.371

É importante que no debate de gênero na disciplina etnobiologia, bem como em outros ramos da ciência, sobretudo nas ciências da natureza, outros fatores podem igualmente influenciar o acesso das mulheres nessas áreas ou, nos casos em que o ingresso chega a acontecer, é preciso nos atentar à permanência, que muitas vezes se torna um desafio. Entre eles, podemos citar a maternidade, uma vez que a capacidade profissional de pesquisadoras que são mães é frequentemente questionada (SILVA et al., 2019). Além disso, é possível que o fator geracional exerça um impacto na participação de mulheres na pesquisa científica, sobretudo aquelas que não ocupam posição de liderança em grupos de pesquisa. Dessa forma, a dificuldade no acesso, na permanência de uma determinada linha de pesquisa, bem como as menores chances de alcançar posições de alta hierarquia, podem ter grande influência no padrão de citação por gênero em determinadas subáreas da etnobiologia.

Atualmente, muito se discute sobre como contribuir para o avanço da etnobiologia enquanto disciplina teórica e, nesse sentido, temos diversos trabalhos, inclusive alguns de cunho cientométrico, que se propõem a contribuir com o avanço dessas questões (ver RAMOS et al. 2012; ALBUQUERQUE, 2013; ALBUQUERQUE et al., 2013; CAMPOS et al., 2016; GAOUE et al, 2017; FERREIRA-JÚNIOR, 2020). Todavia, há ainda uma grande lacuna sobre as questões enfrentadas por etnobiólogas ao redor do mundo. Estudos como o realizado por Silva et al. (2019) e o presente estudo apresentam grande potencial para o desenvolvimento da etnobiologia, pois não se tratam simplesmente de levantamentos e discussões sobre os desafios encontrados por mulheres etnobiólogas, mas devem ser vistos como contribuintes no processo do desenvolvimento da ciência, uma vez que condições igualitárias e ideais de trabalho em pesquisa científica são requisitos básicos para a produção de trabalhos de qualidade capazes de contribuir no avanço da ciência e tecnologia.

\section{CONCLUSÃO}

Nosso trabalho evidenciou que o gênero influenciou o comportamento de citação em pesquisas na área de etnobiologia nos dois últimos anos. Observamos que, independente do continente, autores do gênero masculino citam significativamente mais autores do mesmo gênero e que autoras mulheres citam significativamente mais homens. Com relação às subáreas do conhecimento etnobiológico, autores do gênero masculino citam colegas do mesmo gênero em todas as subáreas, enquanto para autoras do gênero feminino esse padrão foi observado para as subáreas etnobotânica, etnoecologia e etnozoologia. Tais resultados 
CAMPOS, J. L. A.; ALVES, A. S. A.; SANTORO, F. R. As mulheres são menos citadas do que os homens em artigos científicos? Uma análise do comportamento de citação relacionado ao gênero nas pesquisas em etnobiologia. Ethnoscientia v.6 n. 2, especial, 2021. DOI: 10.22276/ethnoscientia.v6i2.371

podem estar associados a um menor número de publicações assinadas por mulheres nos continentes asiáticos e africanos, assim como em todas as subáreas da etnobiologia. Além disso, outras questões já discutidas em estudos anteriores também se relacionam com o padrão de citação observado, como o fato de homens ocuparem maiores cargos de liderança, obterem maior prestígio e possuírem maior acesso a programas de fomento à pesquisa.

A disparidade de gênero e as dificuldades encontradas pelas mulheres cientistas ao redor do mundo atingem não somente a vida dessas mulheres como também o desenvolvimento da ciência, em especial as ciências da natureza. Portanto, à etnobiologia como disciplina em plena ascensão - e composta por um grande corpo de pesquisadoras os resultados obtidos neste trabalho não cabem apenas como fruto de uma investigação fortuita e pontual. Para além disso, devem ser vistos como um instrumento, ainda que incipiente, de discussão e fomento para o subsídio de programas e políticas capazes de estabelecer condições igualitárias de acesso, permanência e desenvolvimento de mulheres na ciência etnobiológica.

\section{REFERÊNCIAS BIBLIOGRÁFICAS}

ABBAS, A.; MUNEER, L. Women empowerment in South Asia: role of women in development of Pakistan. International Journal of Research in Humanities and Social Studies, v.6, n. 2, p. 42-49, 2019.

ALBORNOZ, M.; et al. Las brechas de género en la producción científica Iberoamericana. Papeles del Observatorio, Buenos Aires, Argentina, v. 9, p.31-46, 2018.

ALBUQUERQUE, U. P. How to improve the quality of scientific publications in ethnobiology. Ethnobiology and Conservation, v. 2, n. 4, 2013.

ALBUQUERQUE, U. P. et al. The current status of ethnobiological research in Latin America: gaps and perspectives. Journal of Ethnobiology and Ethnomedicine, v. 9, n.1, 2013.

ALBUQUERQUE, U. P.; RAMOS, M.A.; Medeiros, M.F.T. Experiences of ethnobanists with publication: a fisrt approach. BioScience, v. 61, n. 9, p. 706-712, 2011.

ALVARENGA, L. Bibliometria e arqueologia do saber de Michel Foucault: traços de identidade teórico-metodológica. Ciência da informação, v. 27, n. 3, p. 253-261, 1998.

AYRES, M. et al. BioEstat 5.3: aplicações estatísticas nas áreas das ciências biomédicas. Belém do Pará: Sociedade Civil Mamirauá, 2007.

BRASIL. 2019. Instituto Nacional de Estudos e Pesquisas Educacionais Anísio Teixeira (Inep). Censo da Educação Superior 2018: notas estatísticas. Brasília. Disponível em: 
CAMPOS, J. L. A.; ALVES, A. S. A.; SANTORO, F. R. As mulheres são menos citadas do que os homens em artigos científicos? Uma análise do comportamento de citação relacionado ao gênero nas pesquisas em etnobiologia. Ethnoscientia v.6 n. 2, especial, 2021. DOI: 10.22276/ethnoscientia.v6i2.371

http://download.inep.gov.br/educacao_superior/censo_superior/documentos/2019/censo_da _educacao_superior_2018-notas_estatisticas.pdf. Acesso em: 23 set. 2020.

BEAUDRY, C.; LARIVIÈRE, V. Which gender gap? Factors affecting researchers' scientific impact in science and medicine. Research Policy, n. 45, v .9, p. 1790-1817, 2016.

BORNMANN, L.; DANIEL, H. What do citation counts measure? A review of studies on citing behavior. Journal of Documentation, n. 64, v.1, p. 45-80, 2008.

CAMPOS, J. L. A. et al. Insularity and citation behavior of scientific articles in young fields: the case of ethnobiology. Scientometrics, n. 109, v. 2, p. 1037-1055, 2016.

CHADHURI, S. Women's empowerment in South Asia and Southeast Asia: a comparative analysis. MPRA Paper, 2009.

COTROPIA, C. A.; PETHERBRIDGE, L. Gender disparity in law review citation rates. William \& Mary Law Review, v. 59, n. 3, p. 771-812, 2018.

EUROPEAN UNION. 2019. She figures 2018. Disponível em: https://www.etag.ee/wpcontent/uploads/2019/03/She-Figures-2018-1.pdf. Acesso em 07 out. 2020.

EYSENBACH, G. Citation advantage of open access articles. PLoS Biology, v. 4, n. 5, 2006.

FERREIRA-JÚNIOR, W. S. Reflections on the theoretical advance in ethnobiology: are we pointing to the wrong direction? Ethnobiology and Conservation, v. 9, 2020.

GALINDO, M. Z. Gender in science: the impact of equality policies in scientific institutions and practices: the case of Germany. Cadernos Pagu, v. 47, 2016.

GARGOURI, Y. et al. Self-selected or mandated, open access increases citation impact for higher quality research. PLoS One, v. 5, n. 10, 2010.

GAOUE, O. G. et al. Theories and major hypotheses in ethnobotany. Economic Botany, v. 71, n. 3, p. 269-278, 2017.

HANAZAKI, N. Why are we so attached to the "ethno" prefix in Brazil? Scientometrics, v. 103, n. 2, p. 545-554, 2015.

HOHENDORFF, J. V.; PEREIRA, A. S.; Koller, S. H. Nas "filas de espera": tempo entre submissão e aceitação de manuscritos em periódicos brasileiros de psicologia. Trends in Psychology, v. 24, n. 4, p. 1329-1341, 2016.

IP, N. Y. Career development for women scientists in Asia. Neuron, v. 70, n. 6, p. 10291032, 2011.

KING, M. M. et al. Men set their own cites high: Gender and self-citation across fields and over time. Socius, v. 3, 2017. 
CAMPOS, J. L. A.; ALVES, A. S. A.; SANTORO, F. R. As mulheres são menos citadas do que os homens em artigos científicos? Uma análise do comportamento de citação relacionado ao gênero nas pesquisas em etnobiologia. Ethnoscientia v.6 n. 2, especial, 2021. DOI: 10.22276/ethnoscientia.v6i2.371

LARIVIÈRE, V. et al. Bibliometrics: Global gender disparities in science. Nature News, v. 504, n. 7479, p. 211-213, 2013.

LETA, J. As mulheres na ciência brasileira: crescimento, contrastes e um perfil de sucesso. Estudos Avançados v. 17, n. 49, p. 271-284, 2003.

LORTIE C.J. et al. Publication bias and merit in ecology. Oikos, v. 116, n.7, 1247-1253, 2007.

MALINIAK, D.; POWERS, R.; WALTER, B.F. The gender citation gap in international relations. International Organization, v. 67, n. 4, p. 889-922, 2013.

NARAYAN, L.; SHARMILA, B. Declining women labour force participation in India causes and issues. ZENITH International Journal of Mutidisciplinarity Research, v. 9, n. 4, p. 294-309, 2019.

OKEKE, I. N. et al. Broadening participation in the sciences within and from Africa: purpose, challenges, and prospects. CBE - Life Sciences Education, v. 16, n. 2, 2017.

OSBORN, M. Status and prospects of women in science in Europe. Science, v. 263, n. 5152, p. 1389-1391, 1994.

PROZESKY, H.; MOUTON, J. A gender perspective on career challenges experienced by Africans scientists. South African Journal of Science, v. 115, n. 3-4, p. 1-5, 2019.

$\mathrm{R}$ Development Core Team. 2015. A language and environment for statistical computing. $R$ Foundation for Statistical Computing. Vienna. URL http://www.Rproject.org.

RAMOS, M. A.; MELO, J. G.; ALBUQUERQUE, U. P. Citation behavior in popular scientific papers: What is behind obscure citations? The case of ethnobotany. Scientometrics, v. 92, n. 3, p. 711-719, 2012.

RITTER, M. R. et al. Bibliometric analysis of ethnobotanical research in Brazil (19882013). Acta Botanica Brasilica, v. 29, n. 1, p. 113-119, 2015.

SHAH, T. A.; GUL, S.; GAUR, R. C. Authors self-citation behaviour in the field of Library and Information Science. Aslib Journal of Information Management v. 67, n. 4, p. 458468, 2015.

SILVA, M. R.; HAYASHI, C. R. M.; HAYASHI, M. C. P. I. Análise bibliométrica e cientométrica: desafios para especialistas que atuam no campo. InCID: Revista de Ciência da Informação e Documentação, v. 2, n. 1, 110-129, 2011.

SILVA, T. C. et al. The role of women in brazilian ethnobiology: challenges and perspectives. Journal of Ethnobiology and Ethnomedicine, v. 15, n. 44, p. 1-11, 2019.

SOTERO, M. C. et al. Local and scientific knowledge in the school context: characterization and content of published works. Journal of Ethnobiology Ethnomedicine, v. 16, n. 23, 2020. 
CAMPOS, J. L. A.; ALVES, A. S. A.; SANTORO, F. R. As mulheres são menos citadas do que os homens em artigos científicos? Uma análise do comportamento de citação relacionado ao gênero nas pesquisas em etnobiologia. Ethnoscientia v.6 n. 2, especial, 2021. DOI: 10.22276/ethnoscientia.v6i2.371

TAMALE, S.; OLOKA-ONYANGO, J. Bitches at the academy: gender and academic freedom at the African University. Africa Development, v. 22, n. 1, p. 13-37, 1997.

UNESCO (United Nations Education, Scientific and Cultural Organization). 2018. Women in Science. Unesco Institute for Statistics. Fact Sheet, $\mathrm{n}^{\circ}$ 51. Disponível em: http://uis.unesco.org/sites/default/files/documents/fs51-women-in-science-2018-en.pdf. Acesso em: 05 out. 2020.

VANZ, S. A. S.; CAREGNATO, S.E. 2003. Estudos de citação: uma ferramenta para entender a comunicação científica. Em Questão, v. 9, n. 2, p. 295-307.

WHO (World Health Organization). 2020. Africa's Women in Science. Disponível em: http://158.232.12.119/tdr/research/gender/Women_overview_piece.pdf. Acesso em: 06 out. 2020 . 